\title{
Prevalência e fatores associados a quedas em idosos em um município do Rio de Janeiro
}

Prevalence and factors associated to falls in elderly in a county from Rio de J aneiro, Brazil

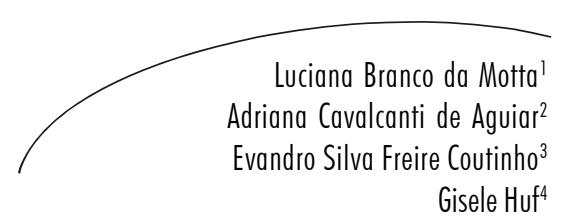

Resumo

Introdução: Estima-se que $50 \%$ das quedas em idosos resultem em algum tipo de lesão. O objetivo deste estudo é estimar a prevalência e identificar fatores associados a quedas em idosos em um município no Rio de Janeiro. Metodologia: Um inquérito foi conduzido em residentes com mais de 60 anos capazes de prestar informações por si, através de um questionário de avaliação multidimensional, aplicado por agentes comunitários treinados. As análises incluíram cálculos de proporções e respectivos intervalos de confiança de 95\%. Resultados: Foram entrevistados 1.064 idosos, em sua maioria mulheres (57\%), com idade média de 71,4 anos. No ano anterior à entrevista, 322 participantes (30,3\% - IC95\% 27,6-33,2) caíram, dos quais 148 $(13,9 \%$ - IC95\% 11,9-16,2) o fizeram pelo menos duas vezes. Algumas associações se evidenciaram: sexo feminino, idade avançada, ser divorciado, morar só, assim como as variáveis indicadoras de más condições de saúde, capacidade funcional e satisfação com a vida . Conclusão: Este estudo ratifica fatores associados a quedas em idosos já conhecidos. O grande desafio deste conhecimento é a proposição de intervenções nos fatores modificáveis e a identificação ativa dos idosos sob risco, objetivando a reabilitação preventiva.

\footnotetext{
Universidade do Estado do Rio de Janeiro. Universidade Aberta da Terceira Idade, Núcleo de Atenção ao Idoso. Rio de Janeiro, RJ, Brasil.

2 Universidade Estácio de Sá. Mestrado Profissional em Saúde da Família. Rio de Janeiro, RJ, Brasil.

3 Fundação Oswaldo Cruz. Escola Nacional de Saúde Pública, Departamento de Epidemiologia. Rio de Janeiro, RJ, Brasil.

${ }_{4}$ Fundação Oswaldo Cruz. Instituto Nacional de Controle de Qualidade em Saúde. Rio de Janeiro, RJ, Brasil.

Fonte de financiamento: $\mathrm{CNPq}$

Correspondência / Correspondence

Luciana Branco da Motta

E-mail: lumotta@uerj.br
}

Palavras-chave: Acidentes por Quedas. Idoso. Fatores de Risco. 


\section{Abstract}

Introduction: Fifty percent of falls in elderly result in some kind of injury. This study aims to assess the prevalence and identify factors associated with falls in aged people in a county of the state of Rio de Janeiro, Brazil. Methods: A survey was conducted with people 60 years-old or more who were able to respond by themselves to a multidimensional assessment questionnaire applied by trained community health agents. We calculated proportions and $95 \%$ confidence intervals. Results: 1,064 individuals were included. They were mostly women $(57 \%)$, with mean age of 71,4 years. 322 participants $(30,3 \%-95 \%$ CI $27,6-33,2)$ fell during the previous year, and $148(13,9 \%$ - 95\%CI 11,9-16,2) fell at least twice. Some associations became evident: female sex, advanced age, being divorced, living alone, as well as indicators of bad health condition, functional capacity and satisfaction with life. Conclusion: This study highlights already known factors associated to falls in the elderly. The major challenge is the proposition of interventions towards modifiable factors and active identification of persons under risk of falling aiming at preventive rehabilitation.

\section{INTRODUÇÃO}

O envelhecimento populacional no Brasil é fato conhecido. ${ }^{1}$ Este processo traz como desafio lidar com agravos como doenças crônicodegenerativas e seu enfrentamento requer práticas em saúde que se voltem para a promoção de saúde, prevenção e identificação precoce de fatores de risco conhecidos, conforme indicado pela Política Nacional de Saúde da Pessoa Idosa (PNSPI). ${ }^{2,3}$

Mais de um terço das pessoas com idade igual ou superior a 65 anos apresenta uma queda ao ano e em muitos casos esta é recorrente. ${ }^{4}$ As quedas podem ser classificadas pela sua frequência, pelas consequências que acarretam, ou de acordo com seu fator de gênese: intrínsecos, extrínsecos e ambientais. ${ }^{5}$ Fatores ambientais se relacionam com riscos do meio ambiente, podendo ocasionar episódio de queda como evento único. Os extrínsecos se relacionam a situações como o uso de múltiplas medicações. ${ }^{5}$ Já os fatores intrínsecos se relacionam a doenças e disfunções do processo de envelhecimento: doenças crônicas, distúrbios do equilíbrio corporal e déficits sensoriais, sendo responsáveis por quedas recorrentes. ${ }^{5}$

Queda é um evento que ocorre em idosos que vivem na comunidade, hospitalizados e institucionalizados. No Brasil, estima-se que o
Key words: Accidental Falls. Aged. Risk Factors. número de quedas em idosos possa ser no mínimo 350 milhões por ano, sendo que $50 \%$ resultariam em algum tipo de lesão, das quais $10 \%$ seriam lesões graves, incluindo fraturas. ${ }^{6}$

As consequências das quedas variam desde pequenas escoriações, passando por graves fraturas, até a morte. Entre 5 a $10 \%$ dos idosos que caem ao ano, e que residem na comunidade, sofrem lesões severas como fraturas, traumatismos cranianos e lacerações sérias, fatores que reduzem mobilidade e independência, com consequente deterioração funcional e maior possibilidade de morte. ${ }^{5}$ Após uma queda de consequência grave, o idoso pode perder ou ter reduzido o seu grau de independência prévio ao evento, pode precisar ser asilado, ou até ir ao óbito. Cerca de metade de idosos hospitalizados por fratura de quadril não recupera a mobilidade anterior ao evento. ${ }^{7}$ Mesmo as quedas sem lesões diretas podem restringir as atividades habituais nesta população devido à dor, ao medo, ou pelo excesso de proteção dos familiares. ${ }^{8}$ Em 2005 , as quedas na população com 60 anos ou mais representaram 2,8\% das internações no Brasil e 3,7\% no Estado do Rio de Janeiro. Em 2004, 0,6\% dos óbitos em idosos foram causados por quedas. ${ }^{8}$

O reconhecimento dos fatores associados à queda, principalmente os que permitem 
intervenção, pode contribuir para a prevenção de sua ocorrência e redução de suas consequências, fomentando a manutenção da autonomia e independência dos idosos, assim como sua qualidade de vida. $\mathrm{O}$ objetivo deste estudo foi estimar a prevalência de queda no último ano e estratificá-la segundo um conjunto de características individuais nos idosos residentes em um município do Estado do Rio de Janeiro, a fim de orientar intervenções preventivas posteriormente junto à Estratégia de Saúde da Família da Secretaria Municipal de Saúde.

\section{METODOLOGIA}

\section{Desenho e local do estudo}

Um estudo seccional foi conduzido em idosos com 60 anos ou mais residentes no Município de Engenheiro Paulo de Frontin entre os meses de setembro e novembro de 2007. O município possui 12.250 habitantes, sendo que $11,78 \%$ têm mais de 60 anos. Este é um município da região centro-sul fluminense com área grande, porém baixa densidade demográfica $\left(85 \mathrm{hab} / \mathrm{Km}^{2}\right)$, predominantemente rural, com longas distâncias entre as localidades como Centro, Sacra Família, Morro Azul, e com transporte intramunicipal pouco eficiente. Seu relevo é acidentado, havendo muitas ladeiras para o deslocamento das pessoas.

\section{Participantes}

Foram selecionados todos os residentes com 60 anos ou mais capazes de prestar as informações solicitadas por si, sem o auxílio de cuidadores ou familiares e capazes de deambular. Os critérios de exclusão foram: incapacidade de responder segundo informação do familiar ou alta dependência e recusa em assinar o termo de consentimento informado.

\section{Instrumento}

O instrumento utilizado foi um questionário de avaliação multidimensional que engloba questões relacionadas a capacidade funcional, com questões fechadas, que teve como base os questionários utilizados por Lima Costa et $\mathrm{al}^{9} \mathrm{e}$ Veras. ${ }^{10}$ Este instrumento foi aplicado no domicílio dos participantes por agentes de saúde comunitários das cinco unidades da Estratégia Saúde da Família do município após treinamento específico. O instrumento foi testado previamente com 30 indivíduos que fizeram parte posteriormente da amostra.

\section{Variáveis}

Foram coletados dados referentes ao perfil sociodemográfico (sexo, idade, estado conjugal, anos de estudo), aos recursos e apoio social (renda mensal, se considera a renda suficiente para as necessidades básicas, se mora só, se existe alguém que possa cuidar do idoso em caso de necessidade, se ele cuida de alguém, se sente só ou isolado a maior parte do tempo), de condições de saúde física (se nos últimos quatro anos esteve internado mais de duas vezes, se percebe alguma dificuldade visual ou auditiva), psicocognitivas (se considera sua saúde boa, se tem pouco interesse ou prazer nas atividades diárias, avaliação da memória comparada a dez anos atrás) e capacidade funcional (se encontra dificuldade para: alimentarse, tomar banho ou ir ao banheiro; correr, levantar peso, fazer esporte ou realizar trabalho pesado; empurrar uma mesa ou fazer trabalho doméstico; subir escada; abaixar-se ou ajoelhar; caminhar mais de um quilômetro e caminhar cerca de 100 metros). A variável de interesse foi definida como a mudança inesperada da posição do corpo, de forma não intencional que faz com que o indivíduo saia de um plano superior e desça a um nível inferior. ${ }^{11} \mathrm{~A}$ pergunta do questionário foi: "O Sr (a) caiu no último ano? Quantas vezes?"

\section{Análise}

As análises descritivas incluíram cálculos de proporções e respectivos intervalos de confiança de $95 \%$. $\mathrm{Na}$ análise bivariada, a prevalência de uma ou mais quedas foi calculada para cada grupo das variáveis intervalos de confiança de $95 \%$ foram calculados e o nível de significância foi testado usando os testes de Qui-quadrado. Os pacotes estatísticos utilizados foram oSPSS, versão12.0 e Epinfo 6,0. 
O Comitê de Ética da Universidade do Estado do Rio de Janeiro aprovou o protocolo do estudo em 2006, e um Termo de Consentimento Livre e Esclarecido foi obtido de todos os participantes.

\section{RESULTADOS}

\section{Características da população}

A população de idosos com 60 anos ou mais do Município de Engenheiro Paulo de Frontin consistia de 1.444 indivíduos na época do estudo. Foram considerados elegíveis 1.145 indivíduos, mas 45 não foram encontrados e 36 recusaram-se a participar. Foram entrevistados 1.064 idosos (93\%) cujo perfil consta da tabela 1 . Estes eram mulheres em sua maioria (57\%), casados e viúvos (81\%), com uma idade média de 71,4 anos. De um modo geral, trata-se de uma população pobre, com poucos anos de escolaridade. Cerca de $90 \%$ dos idosos tem renda entre um e quatro salários mínimos e apenas um terço estudou por mais de quatro anos. Menos de um quinto tinha sobre si a responsabilidade com os cuidados de outra pessoa. Uma vez que não foram considerados elegíveis os idosos realmente incapacitados, entre 30 e $50 \%$ queixavam-se de limitações em alguma capacidade funcional, com exceção das atividades mais elementares, como tomar banho e alimentarse, onde este índice era menor. $\mathrm{Na}$ avaliação das capacidades cognitivas e do humor, $83 \%$ declararam sentir-se amparada em caso de necessidade, mas apesar disso, cerca de $30 \%$ sentiam-se só e $50 \%$ afirmaram ter perdido o interesse por suas atividades. Apenas cerca de um terço considerava sua renda suficiente e sua saúde boa.

Tabela 1 - Ocorrência de queda segundo características dos idosos. Engenheiro Paulo de Frontin, RJ, 2007.

\begin{tabular}{|c|c|c|c|c|}
\hline Variáveis* & $\begin{array}{l}\text { Idosos com uma queda } \\
\% \text { (IC } 95 \% \text { ) }\end{array}$ & $\begin{array}{l}\text { Idosos com + de uma } \\
\text { queda } \\
\% \text { (IC95\%) }\end{array}$ & $\begin{array}{l}\text { Missing } \\
\mathrm{N}\end{array}$ & $\begin{array}{l}\text { Total ** } \\
\text { N (\%) }\end{array}$ \\
\hline \multicolumn{5}{|l|}{ Sexo } \\
\hline Masculino & $12,4(9,6-15,8)$ & $10,3(7,6-13,4)$ & 3 & $458(43)$ \\
\hline \multirow[t]{2}{*}{ Feminino } & $19,4(16,3-22,8)$ & $16,7(13,8-19,9)$ & & $606(57)$ \\
\hline & $\mathrm{p}$-valor $=0,002$ & $\mathrm{p}$-valor $=0,003$ & & \\
\hline Idade média(DP) & $71,4(8,6)$ & $72,9(8,4)$ & 5 & $71,4(8,0)$ \\
\hline Faixa etária & & & 5 & \\
\hline $60-64$ & $18,6(14,0-24,0)$ & $11,9(8,1-16,5)$ & & $253(23,9)$ \\
\hline $65-69$ & $16,2(11,7-21,6)$ & $9,8(6,3-14,4)$ & & $234(22,1)$ \\
\hline $70-74$ & $15,5(10,9-21,1)$ & $17,4(12,5-23,1)$ & & $213(20,1)$ \\
\hline $75-79$ & $15,1(10,1-20,6)$ & $15,1(10,1-20,6)$ & & $212(20,0)$ \\
\hline \multirow[t]{2}{*}{80 ou +} & $16,3(10,7-23,3)$ & $17,7(11,9-24,8)$ & & $147(13,9)$ \\
\hline & $\mathrm{p}$-valor $=0,867$ & $\mathrm{p}$-valor $=0,083$ & & \\
\hline Estado conjugal & & & 7 & \\
\hline Casado & $16,1(12,9-19,7)$ & $10,9(8,2-14,0)$ & & $479(43,3)$ \\
\hline Viúvo & $16,2(12,7-20,2)$ & $15,2(11,8-19,1)$ & & $394(37,3)$ \\
\hline Divorciado & $17,6(10,4-27,0)$ & $24,2(15,8-34,3)$ & & $91(8,3)$ \\
\hline \multirow[t]{2}{*}{ Solteiro } & $17,2(10,2-26,4)$ & $12,9(6,8-21,4)$ & & $93(8,8)$ \\
\hline & $\mathrm{p}$-valor $=0,981$ & $\mathrm{p}$-valor $=0,006$ & & \\
\hline Anos de estudo & & & 23 & \\
\hline Nenhum & $15,7(11,7-20,4)$ & $14,6(10,7-19,3)$ & & $287(27,6)$ \\
\hline $1-3$ & $16,9(13,4-20,8)$ & $13,3(10,1-16,9)$ & & $415(39,9)$ \\
\hline $4-7$ & $16,4(11,8-21,8)$ & $15,1(10,7-20,3)$ & & $232(22,3)$ \\
\hline \multirow[t]{2}{*}{8 ou +} & $15,0(8,8-23,1)$ & $12,1(6,6-19,9)$ & & $107(10,3)$ \\
\hline & p-valor $=0,956$ & $\mathrm{p}$-valor $=0,882$ & & \\
\hline
\end{tabular}


Tabela 1 - Ocorrência de queda segundo características dos idosos. Engenheiro Paulo de Frontin, RJ, 2007. (continuação)

\begin{tabular}{|c|c|c|c|c|}
\hline Variáveis* & $\begin{array}{l}\text { Idosos com uma queda } \\
\% \text { (IC } 95 \%)\end{array}$ & $\begin{array}{l}\text { Idosos com + de uma } \\
\text { queda } \\
\%(\text { IC } 95 \%)\end{array}$ & $\begin{array}{l}\text { Missing } \\
\mathrm{N}\end{array}$ & $\begin{array}{l}\text { Total } * * \\
N(\%)\end{array}$ \\
\hline Renda mensal (sm) & & & 83 & \\
\hline sem renda & $15,2(6,3-28,9)$ & $10,9(3,6-23,6)$ & & $46(4,7)$ \\
\hline$<1$ & $27,8(9,7-53,5)$ & $5,6(0,1-27,3)$ & & $18(1,8)$ \\
\hline entre 1 e 1,9 & $16,6(13,8-19,7)$ & $16,0(13,3-19,0)$ & & $650(66,3)$ \\
\hline entre 2 e 3,9 & $16,6(11,8-22,3)$ & $11,4(7,4-16,4)$ & & $211(21,5)$ \\
\hline$\geq 4$ & $\begin{array}{l}17,9(8,9-30,4) \\
\text { p-valor }=0,791\end{array}$ & $\begin{array}{l}5,4(1,1-14,7) \\
\text { p-valor }=0,081\end{array}$ & & $56(5,7)$ \\
\hline Mora só & & & 4 & \\
\hline Sim & $13,0(8,7-18,5)$ & $19,0(13,8-25,1)$ & & $200(18,9)$ \\
\hline Não & $\begin{array}{l}17,2(14,7-19,9) \\
\text { p-valor }=0,148\end{array}$ & $\begin{array}{l}12,8(10,3-15,2) \\
\text { p-valor }=0,022\end{array}$ & & $860(81,1)$ \\
\hline$>2$ internações nos últimos 4 anos & & & 34 & \\
\hline $\operatorname{sim}$ & $21,0(15,8-27,1)$ & $27,1(21,3-33,6)$ & & $214(20,8)$ \\
\hline não & $\begin{array}{l}15,4(13,0-18,1) \\
\text { p-valor }=0,050\end{array}$ & $\begin{array}{l}10,4(804-12,7) \\
\text { p-valor }=0,000\end{array}$ & & $816(79,2)$ \\
\hline Dificuldade visual & & & 17 & \\
\hline Sim & $19,5(16,3-23,1)$ & $18,2(15,1-21,7)$ & & $543(51,9)$ \\
\hline Não & $\begin{array}{l}13,1(10,3-16,3) \\
\text { p-valor }=0,005\end{array}$ & $\begin{array}{l}9,5(7,1-12,4) \\
\text { p-valor }=0,000\end{array}$ & & $504(48,1)$ \\
\hline Dificuldade auditiva & & & 9 & \\
\hline Sim & $20,2(16,0-24,9)$ & $17,2(13,3-21,7)$ & & $337(31,9)$ \\
\hline Não & $\begin{array}{l}14,6(12,1-17,4) \\
\text { p-valor }=0,023\end{array}$ & $\begin{array}{l}12,4(10,1-15,0) \\
\text { p-valor }=0,035\end{array}$ & & $718(68,1)$ \\
\hline $\begin{array}{l}\text { Dificuldade para alimentar-se, tomar } \\
\text { banho ou ir ao banheiro }\end{array}$ & & & 8 & \\
\hline Sim & $27,8(16,4-31,4)$ & $28,6(21,7-37,0)$ & & $133(12,6)$ \\
\hline Não & $\begin{array}{l}14,8(12,6-17,3) \\
\text { p-valor }=0,000\end{array}$ & $\begin{array}{l}11,7(9,7-13,9) \\
\text { p-valor }=0,000\end{array}$ & & $923(87,4)$ \\
\hline $\begin{array}{l}\text { Dificuldade para correr, levantar } \\
\text { peso, fazer esporte ou realizar } \\
\text { trabalho pesado }\end{array}$ & & & 6 & \\
\hline Sim & $17,5(14,5-20,8)$ & $17,1(14,1-20,4)$ & & $578(54,6)$ \\
\hline Não & $\begin{array}{l}15,2(12,1-18,7) \\
\text { p-valor }=0,322\end{array}$ & $\begin{array}{l}10,2(7,6-13,3) \\
\text { p-valor }=0,001\end{array}$ & & $480(45,4)$ \\
\hline $\begin{array}{l}\text { Dificuldade para empurrar uma mesa } \\
\text { ou fazer trabalho doméstico }\end{array}$ & & & 8 & \\
\hline Sim & $17,8(14,4-21,6)$ & $18,9(15,4-22,7)$ & & $461(43,7)$ \\
\hline Não & $\begin{array}{l}15,5(12,6-18,6) \\
\text { p-valor }=0,312\end{array}$ & $\begin{array}{l}10,3(7,9-13,0) \\
\text { p-valor }=0,000\end{array}$ & & $595(56,3)$ \\
\hline Dificuldade para subir escada & & & 13 & \\
\hline Sim & $19,6(16,2-23,3)$ & $20,5(17,1-24,3)$ & & $511(48,6)$ \\
\hline Não & $\begin{array}{l}13,3(10,6-16,5) \\
\text { p-valor }=0,006\end{array}$ & $\begin{array}{l}8,0(5,8-10,6) \\
\text { p-valor }=0,000\end{array}$ & & $540(51,4)$ \\
\hline $\begin{array}{l}\text { Dificuldade para abaixar-se ou } \\
\text { ajoelhar }\end{array}$ & & & 8 & \\
\hline $\operatorname{Sim}$ & $22,5(18,4-27,1)$ & $21,7(17,6-26,2)$ & & $373(35,3)$ \\
\hline Não & $\begin{array}{l}13,2(10,7-15,9) \\
\text { p-valor }=0,000\end{array}$ & $\begin{array}{l}9,7(7,5-12,1) \\
\text { p-valor }=0,000\end{array}$ & & $683(64,7)$ \\
\hline Dificuldade para caminhar $+1 \mathrm{~km}$ & & & 6 & \\
\hline Sim & $19,8(16,4-23,7)$ & $19,2(15,8-23,0)$ & & $484(45,7)$ \\
\hline Não & $\begin{array}{l}13,2(10,6-16,3) \\
\text { p-valor }=0,004\end{array}$ & $\begin{array}{l}9,4(7,1-12,1) \\
\text { p-valor }=0,000\end{array}$ & & $574(54,3)$ \\
\hline
\end{tabular}


Tabela 1 - Ocorrência de queda segundo características dos idosos. Engenheiro Paulo de Frontin, RJ, 2007. (continuação)

\begin{tabular}{|c|c|c|c|c|}
\hline \multirow[t]{2}{*}{ Variáveis* } & $\begin{array}{l}\text { Idosos com uma queda } \\
\% \text { (IC } 95 \% \text { ) }\end{array}$ & $\begin{array}{l}\text { Idosos com + de uma } \\
\text { queda }\end{array}$ & Missing & Total $* *$ \\
\hline & & $\%(\mathrm{IC} 95 \%)$ & $\mathrm{N}$ & $\mathrm{N}(\%)$ \\
\hline \multicolumn{3}{|l|}{ Dificuldade para caminhar $100 \mathrm{~m}$} & 6 & \\
\hline $\operatorname{Sim}$ & $21,0(16,8-25,6)$ & $21,0(16,8-25,6)$ & & $348(32,9)$ \\
\hline \multirow[t]{2}{*}{ Não } & $13,9(11,5-16,7)$ & $10,6(8,4-13,1)$ & & $710(67,1)$ \\
\hline & $\mathrm{p}$-valor $=0,003$ & $\mathrm{p}$-valor $=0,000$ & & \\
\hline \multicolumn{2}{|l|}{ Considera a saúde boa } & & 4 & \\
\hline Sim & $14,2(10,8-18,2)$ & $7,0(4,6-10,0)$ & & $373(35,2)$ \\
\hline \multirow[t]{2}{*}{ Não } & $17,6(14,8-20,7)$ & $17,8(15,0-20,8)$ & & $687(64,8)$ \\
\hline & $\mathrm{p}$-valor $=0,153$ & $\mathrm{p}$-valor $=0,000$ & & \\
\hline \multicolumn{2}{|l|}{$\begin{array}{l}\text { Comparado com } 10 \text { anos atrás, a } \\
\text { memória está pior }\end{array}$} & & 14 & \\
\hline & $17,5(14,4-21,0)$ & $18,3(15,1-21,9)$ & & $525(50,0)$ \\
\hline \multirow{2}{*}{ Não } & $15,4(12,4-18,8)$ & $9,5(7,1-12,4)$ & & $525(50,0)$ \\
\hline & $\mathrm{p}$-valor $=0,360$ & $\mathrm{p}$-valor $=0,000$ & & \\
\hline \multicolumn{2}{|l|}{ Considera sua renda suficiente } & & 40 & \\
\hline Sim & $13,0(9,6-17,0)$ & $11,2(8,0-15,1)$ & & $339(33,1)$ \\
\hline \multirow[t]{2}{*}{ Não } & $18,4(15,6-21,5)$ & $15,5(12,8-18,4)$ & & $685(66,9)$ \\
\hline & p-valor $=0,028$ & $\mathrm{p}$-valor $=0,065$ & & \\
\hline \multicolumn{2}{|l|}{ É cuidado se necessário } & & 14 & \\
\hline $\operatorname{Sim}$ & $15,5(13,1-18,0)$ & $12,5(10,3-14,8)$ & & $867(82,6)$ \\
\hline \multirow[t]{2}{*}{ Não } & $19,7(14,2-6,2)$ & $21,3(15,6-28,0)$ & & $183(17,4)$ \\
\hline & $\mathrm{p}$-valor $=0,159$ & $\mathrm{p}$-valor $=0,002$ & & \\
\hline \multicolumn{2}{|l|}{ Cuida de alguém } & & 22 & \\
\hline $\operatorname{Sim}$ & $17,2(12,1-23,3)$ & $16,7(11,7-22,7)$ & & $192(18,4)$ \\
\hline \multirow[t]{2}{*}{ Não } & $16,4(13,9-19,0)$ & $13,4(11,2-15,2)$ & & $850(81,6)$ \\
\hline & $\mathrm{p}$-valor $=0,778$ & $\mathrm{p}$-valor $=0,240$ & & \\
\hline \multicolumn{2}{|l|}{ Sente-se só ou isolado } & & 8 & \\
\hline $\operatorname{Sim}$ & $21,4(17,1-26,1)$ & $22,8(18,5-27,7)$ & & $337(31,9)$ \\
\hline \multirow[t]{2}{*}{ Não } & $14,2(11,7-16,9)$ & $9,7(7,7-12,1)$ & & $719(68,1)$ \\
\hline & p-valor $=0,003$ & $\mathrm{p}$-valor $=0,000$ & & \\
\hline $\begin{array}{l}\text { Tem sentido pouco interesse em suas } \\
\text { atividades }\end{array}$ & & & 20 & \\
\hline $\operatorname{Sim}$ & $19,7(16,4-23,4)$ & $18,1(14,9-21,6)$ & & $537(51,4)$ \\
\hline Não & $13,0(10,2-16,3)$ & $9,5(7,1-12,3)$ & & $507(48,6)$ \\
\hline & $\mathrm{p}$-valor $=0,003$ & $\mathrm{p}$-valor $=0,000$ & & \\
\hline
\end{tabular}

*\% calculados na linha

$* \%$ calculados na coluna

Distribuição das quedas segundo

características dos idosos

No ano anterior à entrevista, 322 participantes (30,3\% - IC95\% 27,6-33,2) relataram a ocorrência de quedas, dos quais 148 (13,9\% -IC95\% 11,9$16,2)$ caíram duas ou mais vezes, uma média de 1,94 quedas $(\mathrm{DP}=1,3)$. Três pessoas não puderam ou não souberam responder a esta pergunta.
A ocorrência de uma ou mais de uma queda no último ano também foi estimada segundo diferentes características da população de idosos. Algumas associações apenas se tornaram evidentes para o grupo com duas ou mais quedas. Entre as variáveis sociodemográficas, apenas o sexo feminino apresenta uma prevalência mais elevada tanto de uma, quanto de duas ou mais quedas no último ano. A idade avançada, ser divorciado e 
morar só apresentaram uma prevalência aumentada apenas para duas ou mais quedas no ano anterior à entrevista. Praticamente todas as variáveis indicadoras de condições de saúde e capacidade funcional estiveram claramente associadas à maior prevalência de quedas: quanto piores essas condições, maior a prevalência de uma ou mais de uma queda. Estas associações são mais contundentes, tanto clínica quanto estatisticamente, quando se trata de duas ou mais quedas. Os idosos que caíram apresentavam maior dificuldade para realizar tarefas do dia a dia e referiam maiores dificuldades visuais, auditivas e de memória. Igualmente, as variáveis psicocognitivas, indicadoras de um estado de satisfação com suas condições e consigo próprio, se mostraram associadas com ocorrência de quedas e estas diferenças são significativamente maiores para duas ou mais quedas.

\section{DISCUSSÃO}

O presente estudo mostrou que a ocorrência de quedas no último ano na população estudada é semelhante ao que vem sendo relatado na literatura nacional e internacional. ${ }^{5,6,7,12} \mathrm{O}$ estudo mostrou ainda que a distribuição das quedas não é homogênea, mas que há grupos onde esse acidente é mais comum. Como em outros estudos, nossos resultados mostram uma associação entre gênero, idade avançada, ser separado/viúvo, ter baixo nível socioeconômico, a autopercepção de saúde e satisfação com renda, a capacidade em executar as atividades cotidianas como variáveis associadas a um maior risco de quedas e que permitem intervenções preventivas em todos os seus níveis de ação. ${ }^{712-15}$ No estudo de Fabrício et al., ${ }^{15}$ 54\% das quedas apresentaram como causa um ambiente inadequado, sendo identificadas questões como piso escorregadio, objetos jogados no chão, subir em objetos para alcançar algo, trombar com outras pessoas, degrau e queda da cama, o que enfatiza a possibilidade de ações educativas a serem implementadas em uma abordagem preventiva.

Nosso estudo distinguiu fatores associados à ocorrência de uma queda e a duas ou mais quedas no ano anterior. É possível supor que as pessoas que caem apenas uma vez não compartilham dos mesmos mecanismos subjacentes às quedas múltiplas. Assim, esta distinção pode revelar fatores de risco associados aos principais mecanismos capazes de causar quedas recorrentes.

A principal vantagem deste estudo é basear-se no censo de uma população, com baixo índice de perdas, apenas $7 \%$. No entanto, o desenho de corte transversal pode estar sujeito à causalidade reversa, isto é, não é possível garantir que os fatores associados às quedas sejam causa e não consequência desta experiência. Isto é menos importante no caso de condições sociodemográficas, mas sofrer uma queda está relacionado a limitações físicas e consequências psíquicas, podendo aumentar a sensação de desamparo. Nesse sentido, optamos por apenas indicar variáveis associadas com as quedas, sem fazer inferências causais.

O critério de elegibilidade observado, com a exclusão de indivíduos incapazes de deambular ou de prestar informações por si, foca este estudo numa amostra considerada saudável. Por essas razões, nossos achados tendem a estimar o melhor cenário possível. Mesmo assim, as taxas de queda estimadas por este estudo estão em consonância com a prevalência de queda em idosos em comunidades relatada em outros estudos. ${ }^{7,12}$

A abordagem do idoso com quedas recorrentes é um desafio para os profissionais de saúde e para o Sistema Único de Saúde. As quedas recorrentes estão vinculadas habitualmente a múltiplos fatores tanto intrínsecos, como extrínsecos e/ou ambientais que, atuando de forma sinérgica, propiciam a recorrência dos eventos e apontam para a complexidade da intervenção necessária. Vários protocolos de avaliação e intervenção vêm sendo estudados ${ }^{5,16-18}$ em populações idosas na comunidade visando à redução do risco. Todos incluem ações visando à identificação dos fatores relacionados (e suas correções cabíveis), como a hipotensão postural, o uso de medicações, a avaliação de equilíbrio e marcha, o déficit visual, a força muscular e o meio ambiente. 
A maioria dos idosos que vivem em Engenheiro Paulo de Frontin é acompanhada pela Estratégia Saúde da Família. Sua característica de capilaridade permite que equipes tenham acesso e possam influenciar o ambiente onde os idosos vivem. Sua proposta de acompanhamento preventivo da saúde demanda que ações específicas para a melhora destes fatores de risco sejam implementadas em consonância com a PNSPI. ${ }^{2}$

Agregar qualidade aos anos adicionais vividos é o objetivo maior da Gerontologia e Geriatria. $O$ efeito de programas para melhoria das condições físicas e psicológicas em ensaios clínicos controlados para avaliar intervenções capazes de prevenir quedas são importantes fontes de informação para o desafio de reduzir a prevalência do evento e suas consequências. Estudos que avaliem a eficácia das intervenções sobre fatores de risco já identificados devem ser buscados na população e suas abordagens implementadas quando possível.

\section{CONCLUSÃO}

Este estudo evidencia e ratifica alguns fatores associados a quedas em pessoas idosas, já apontados por estudos anteriores. $\mathrm{O}$ grande desafio é a implementação de medidas necessárias para seu controle, que dependem do envolvimento de vários agentes. Os profissionais de saúde, em especial os da Atenção Primária deveriam ser treinados e sensibilizados para a questão, instrumentalizados na avaliação destes indivíduos e condução das intervenções, com especial atenção à promoção da saúde e educação popular. Noções de risco ambiental, no domicílio ou na comunidade, e atitudes de risco, como uso de calçados inadequados, deveriam fazer parte do trabalho de promoção e educação da população pela Estratégia Saúde da Família. A identificação ativa do idoso com quedas recorrentes também deve ser realizada de forma ativa, pois este é considerado um idoso em risco. A possibilidade de reabilitação dos déficits visuais, do equilíbrio e marcha e da força muscular devem ser prioridade para que o idoso possa manter-se ativo na comunidade. Simplesmente agregar anos de vida não faz sentido sem que haja esperança de que sejam vividos com qualidade.

\section{AGRADECIMENTOS}

Às alunas bolsistas que participaram do trabalho; e aos agentes comunitários de saúde do município pela colaboração. 


\section{REFERÊNCIAS}

1. Lima-Costa MF, Veras RP. Saúde pública e envelhecimento. Cad Saúde Pública 2003; 19(3): 700-1.

2. Brasil. Ministério da Saúde. Portaria n ${ }^{\circ} 2528$ de 19 de outubro de 2006. Aprova Política Nacional da Saúde da Pessoa Idosa. Pub Diário Oficial [da] República Federativa do Brasil, [2006 out. 20].p.142-8.

3. Veras RP, Parayba MI. O anacronismo dos modelos assistenciais para os idosos na área da saúde: desafios para o setor privado. Cad Saúde Pública 2007; 23(10): 2479-89.

4. Tinetti ME. Preventing Falls in Elderly Persons. N Engl J Med 2003 jan; 348(1): 42-9.

5. American Geriatrics Society; British Geriatrics Society; American Academy of Orthopaedic Surgeons Panel on Falls Prevention. Guideline for the prevention of falls in older persons. J Am Geriatr Soc 2001 may; 49(5): 664-72.

6. Anderson MIP. Quedas seguidas de fraturas e hospitalizações em idosos: freqüência, circunstâncias e fatores de risco. [tese]. Rio de Janeiro: Instituto de Medicina Social da Universidade do Estado do Rio de Janeiro; 2003.

7. Perracini MR, Ramos LR. Fatores associados a quedas em uma coorte de idosos residentes na comunidade. Rev Saúde Pública 2002; 36(6): 709-16.

8. Ribeiro AP, et al. A influência das quedas na qualidade de vida de idosos. Cien Saude Colet 2008 jul/ago; 13(4): 1265-73.

9. Lima-Costa MF, Barreto SM, Giatti L. Condições de saúde, capacidade funcional, uso de serviços de saúde e gastos com medicamentos da população idosa brasileira: um estudo descritivo baseado na Pesquisa Nacional por
Amostra de Domicílios. Cad Saúde Pública 2003 mai/jun; 19(3): 735-43.

10. Veras RP. Em busca de uma assistência adequada à saúde do idoso: revisão da literatura e aplicação de um instrumento de detecção precoce e de previsibilidade de agravos. Cad Saúde Pública 2003 mai./jun; 19(3): 705-15.

11. Brown LA, Shumway-Cook A, Woollacott MH. Attentional demands and postural recovery: the effects of aging. J Gerontol A Biol Sci Med Sci 1999 apr; 54(4): M165-71.

12. Siqueira FV, et al. Prevalência de quedas em idosos e fatores associados. Rev Saúde Pública 2007; 41(5): 749-56.

13. Lawlor DA, Patel R, Ebrahim S. Association between falls in elderly women and chronic diseases and drug use: cross sectional study. BMJ 2003 sep; 327(7417): 712-7.

14. Leipzing RM, Cumming RG, Tinetti ME. Drugs and falls in older people: a systematic review and meta-analysis: I. Psychotropic drugs. J Am Geriatr Soc 1999 jan; 47(1): 30-9.

15. Fabrício SCC, Rodrigues RAP, Costa Junior ML. Causas e conseqüências de quedas de idosos atendidos em hospital público. Rev Saúde Pública 2004; 38(1): 93-9.

16. Chang JT, et al. Interventions for the prevention of falls in older adults: systematic review and meta-analysis of randomized clinical trials. BMJ 2004 mar; 328(7441): 680.

17. Feder $\mathrm{G}$, et al. Guidelines for the prevention of falls in people over 65. BMJ 2000 oct; 321(7267): 1007-11.

18. Tinetti ME, et al. A multifactorial intervention to reduce the risk of falling among elderly people living in the community. N Engl J Med 1994; 331(13): 821-7. 
